\title{
Proper mask and glove etiquette for outpatient clinicians and the general public
}

\section{Background on COVID-1 9 infectivity}

It is no doubt that the novel 2019 Coronavirus strain, also known as COVID-19 or SARS CoV-2, has created an unprecedented pandemic. This virus is being studied with daily groundbreaking developments. A recent article found COVID-19 has been found to remain viable in aerosols for 3 hours, similar to that observed with SARS-CoV-1. ${ }^{1}$ COVID-19 was also found to survive on surfaces such as plastic and stainless steel for up to 72 hours after application. ${ }^{1}$ This new research indicates that the COVID-19 has the potential of being transmitted through the air as aerosols and through touching surfaces as a fomite, highlighting the importance of wearing face masks and gloves in the clinical and public setting, especially in outpatient settings and in public areas where there is constant and frequent interaction and movement of people.

Further research revealed that COVID-19 can live longer on smooth surfaces. Specifically, the longest the virus lived on glass and banknotes, such as money, was 4 days. ${ }^{2}$ COVID-19 remained on stainless steel, plastic and strikingly on the outer layer of a surgical mask for 7 days. $^{2}$ However, COVID-19 is susceptible to standard disinfection methods, such as ethanol and household bleach. ${ }^{2}$

In light of these findings, outpatient medical care will have unique challenges in comparison to inpatient care. Some examples include higher patient turnover and a greater number of patients with chronic medical conditions who had been waiting to come into clinics until shelter-in-place rules were relaxed. With the widespread use of masks and gloves by the general public and the increase in outpatient clinic visits, we felt it important to discuss glove and mask etiquette for practitioners in the outpatient setting.

\section{Mask and glove etiquette for the practitioner and healthcare staff}

Given the new research findings that COVID-19 survives on the outside of surgical masks, we urge the following guidelines when training practitioners and healthcare staff, especially since masks are likely to be reused throughout the entire day. Our guidelines for proper mask, glove, and disinfection etiquette for the general public and healthcare workers are summarized in Table 1.

Mask etiquette: All healthcare professionals and staff should be cautious about adjusting surgical masks unconsciously with our gloved hands and then touching patients. If a mask is readjusted, the hand or glove should be sanitized with an appropriate sanitary method before proceeding with the visit.

Glove etiquette: Gloves should not be used to touch patients and then touch other items in the room such as keyboards or medical equipment. Gloves should be removed after touching patients and gloves do not need to be worn at all times except as necessary to perform exams. Because COVID-19 has a respiratory route of entry and not a cutaneous route of entry, frequent hand sanitation should be sufficient. Dermatologists frequently perform facial exams with touching of the
Volume 4 Issue 4 - 2020

\author{
Alexis E Carrington, ${ }^{1,4}$ Shiv Sudhakar,' Hadar \\ Lev-Tov,' Danielle Tartar,' Raja Sivamani' $1,2,3,4,5$ \\ 'Department of Dermatology, University of California-Davis, \\ USA \\ ${ }^{2}$ Department of Biological Sciences, California State University, \\ USA \\ ${ }^{3}$ College of Medicine, California Northstate University, USA \\ ${ }^{4}$ Pacific Skin Institute, USA \\ ${ }^{5}$ Zen Dermatology, USA
}

Correspondence: Raja Sivamani, Department of Dermatology, University of California-Davis, USA, Tel 916-734-6III, Email raja.sivamani.md@gmail.com

Received: July 08, 2020 | Published: July 202020

face to carefully assess for texture signs of actinic keratoses or skin cancers. Gloves should be considered but hand sanitation should be sufficient if a gloveless exam of the face is necessary. Conversation should be limited during the period of the close facial exam when the patient's mask is removed to limit asymptomatic respiratory spread of the coronavirus.

Disinfection: All frequently touched surfaces should be sanitized in between patient visits such as the medical chair and any tables in the rooms. Other frequently touched surfaces such as keyboards and doorknobs should be sanitized as well.

\section{Mask and glove etiquette for general public}

Now that some states in the United States, as well as other countries around the world, are requiring their citizens to wear masks out in public, it is important to highlight proper mask and glove use in the general public. The general public may develop a false sense of security when wearing gloves that may paradoxically increase the risk for transmission if the gloves are not used properly. Patients and the general public should be educated that COVID-19 is a respiratory infection and that gloves should only be worn when touching potentially infected surfaces or when caring for another. Also, patients should be reminded not to touch surfaces like doorknobs after touching their mask or face with gloves. The gloves need to be removed and the focus should be on hand sanitation rather than use of gloves. Physicians and practitioners may serve as an important source of education regarding glove etiquette and we recommend including glove etiquette recommendations as part of aftercare visit summaries.

The CDC has not made official recommendations to wear gloves in public, ${ }^{3,4}$ however, given that COVID-19 can spread on fomites, it is prudent to sanitize after touching public surfaces or handling money or exchanging credit cards during purchasing. Cashless transactions that do not require any physical exchange would be preferred. 
Table I This figure outlines our recommendations for proper mask, glove and disinfectant etiquette for the general public and health care providers, especially in the outpatient setting.

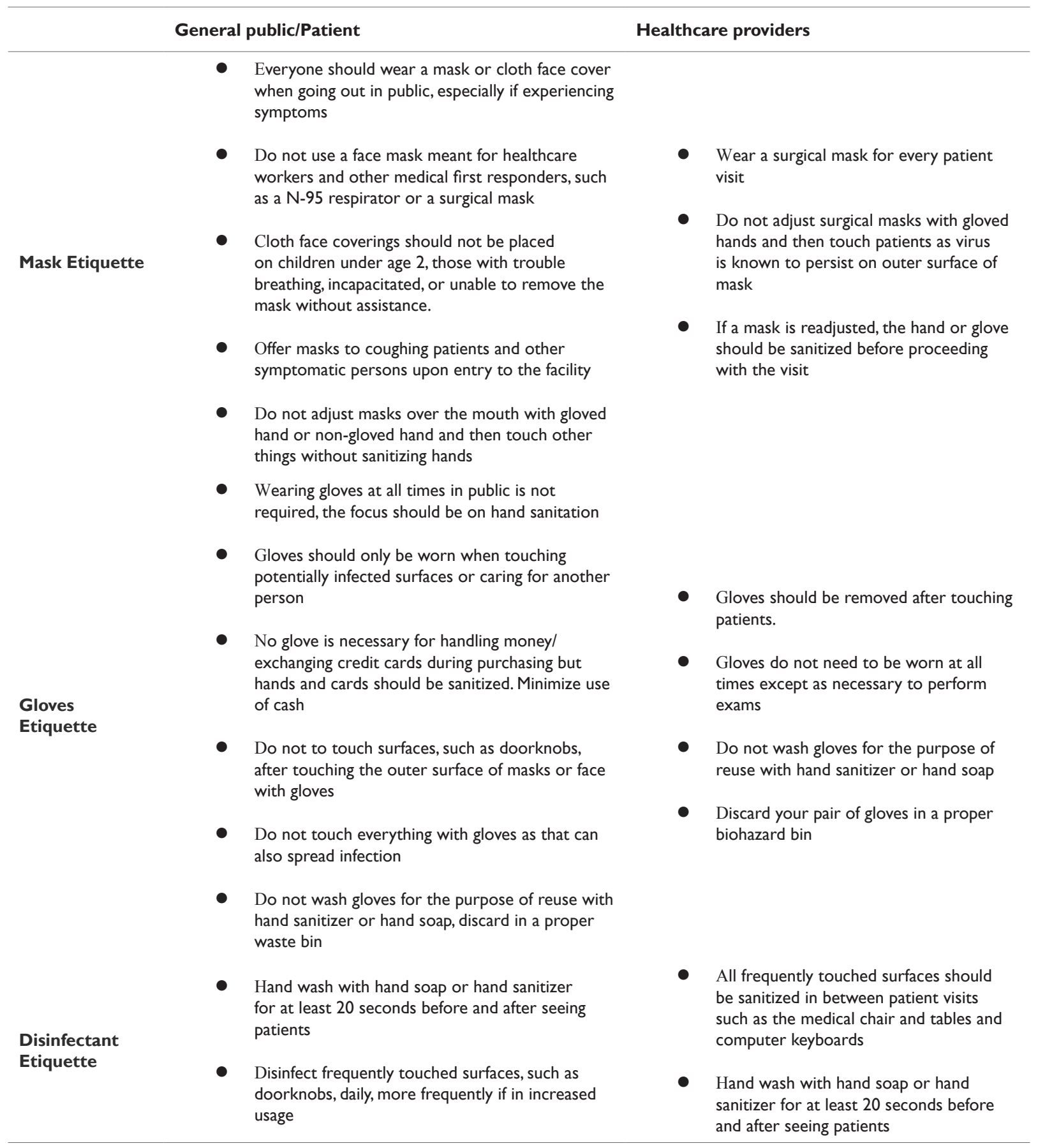

\section{Conflits of interest}

The author declares that there is no conflicts of interest.

\section{Acknowledgments}

None.

\section{Funding}

None.

\section{References}

1. N van Doremalen, Trenton Bushmaker, Dylan H Morris, et al. Aerosol and Surface Stability of SARS-CoV-2 as Compared with SARSCoV-1. N Engl J Med. 2020;382(16):1564-1567.

2. Chin, A. Julie C, Mahen P, et. al. Stability of SARS-CoV-2 in different environmental conditions. The Lancet Microbe. 2020.

3. Greenhalgh T, et. al. Face masks for the public during the covid-19 crisis. BMJ. 2020;369:m1435.

4. Center for Disease Control. Guide to Infection Prevention for Outpatient Settings: Minimum Expectations for Safe Care. Center for Disease Control. 2016. 